\title{
InCidental Discovery of Carotid ARTery Dissection as a CaUSe of Horner's Syndrome and Headache in a Patient Presenting for Follow-Up Of LUMBar EpIDURAL Steroid InJeCtion: A CASE Report WITH A THREe YeAR Follow-UP
}

\author{
Sukdeb Datta, MD, and Kenneth D. Candido, MD
}

Objective : To describe management challenges and follow-up data for three years in a case with spontaneous carotid artery dissection (SCAD).

Case Report : We present a case of SCAD that presented with Horner's syndrome and headache. The patient developed the SCAD, Horner's syndrome and headache one month after a lumbar epidural steroid injection for radicular pain. Three years follow-

Spontaneous carotid artery dissection (SCAD) has been associated with a number of clinical syndromes including transient ischemic attack (TIA), cerebral vascular accident (CVA), cranial neuropathies and migraine headache. This case report presents an unusual presentation of SCAD that included cluster headache and Horner's syndrome in a patient who presented to our pain clinic for follow -up of lumbar epidural steroid injection (LESI) for radicular low back pain.

\section{Case Report}

A 44-year-old Caucasian woman presented to the pain clinic for evaluation and treatment of radicular low back pain. Workup revealed a bulging disk at the L34 level for which she received a lumbar epidural steroid injection (LESI) using $80 \mathrm{mg}$

From VA Tennessee Valley Healthcare System, Nashville, Tennessee and Northwestern University Hospital, , chicago, Illinois

Address Correspondence: Sukdeb Datta, MD, Director, Pain Management Center, VA Tennessee Valley Healthcare System, Nashville Campus, $131024^{\text {th }}$ Avenue South, Nashville, TN 37212

Disclaimer: There was no external funding in preparation of this manuscript.

Funding: Department of Anesthesiology, Cook

County Hospital, Chicago, IL and University of Cin-

cinnati, Cincinnati, $\mathrm{OH}$

Conflict of Interest: None

Acknowledgement:

Manuscript received on 02/04/04

Revision submitted on $04 / 07 / 04$

Accepted for publication on $05 / 10 / 04$ up experience with therapeutic challenges is described.

Conclusion : A life threatening cause of headache is described which occurred one month after receiving an uneventful lumbar epidural steroid injection. Despite no carotid arterial luminal flow restriction and anticoagulation, the headache and Horner's syndrome persisted for three years. Stellate ganglion blocks were able to relieve both neck pain and headache temporarily. In patients with new onset headache and Horner's syndrome SCAD must be ruled out. If SCAD is detected, appropriate therapy including anticoagulation must be instituted immediately, since the potential for continued dissection or embolization remain significant.

Keywords: Headache, spontaneous carotid artery dissection (SCAD), lumbar epidural steroid injection methylprednisolone acetate in $2 \mathrm{ml}$ of $1 \%$ lidocaine hydrochloride with resolution of the radiculopathy pain (VAS diminished from $8 / 10$ to $1 / 10$ at 48 hours). Two months later, she came to the pain clinic for follow - up at which time we noted that she had developed a right-sided Horner's syndrome. We elicited a history of her having "bumped" her head in a supermarket one week following the LESI. The following day, she noted an intense headache confined to the right side of her face and cranium. The patient denied concomitant photophobia, nausea, vomiting, neurological symptoms or visual scintillation. The headache worsened over the next two days. She then developed a drooping of her eyelid. Her primary care physician prescribed Acetaminophen and Tramadol for her headache, which afforded no significant relief. As the headache got progressively worse over the next few weeks, she visited a neurologist who diagnosed cluster headaches and began an empirical regimen of prednisone and verapamil, also without reduction of her symptomatology. The patient was an office manager, had a 30 pack-year history of smoking, and drank an occasional alcoholic beverage on weekends. She was otherwise healthy and active, with no significant past medical history. Past surgical history included three unremarkable gynecological laparoscopies and a hemorrhoidectomy. Family history was significant in that her mother had died at age 33 years secondary to arteriosclerosis and cerebral vascular accident. Physical exam revealed a BP of 123/60-mm hg with a pulse of 72 beats per minute. A right-sided Horner's syndrome with ptosis, miosis, enophthalmos and anhidrosis, along with right-sided conjunctival injection, was obvious and greatly distressed the patient. The remainder of the physical examination was essentially unremarkable, including cranial nerve assessment. Of note, the carotid arterial pulses were normal and no bruits were auscultated.

Our workup included computed tomography (CT) scans of the head and neck and chest X-ray, which all failed to identify any pathology. A magnetic resonance angiogram (MRA) (Fig 1) revealed a dissection of the right internal carotid artery (ICA) with an intimal flap. Ultrasound of neck confirmed the MRA findings, which showed dissection in the right ICA and right common carotid artery (CCA) without significant narrowing of the ICA or the CCA.

Differential diagnosis for the spontaneous dissection of internal carotid artery included vasculitis and connective tissue disorders. Blood was sent for serum complement levels and rheumatoid factor, which were within normal limits. The patient was anticoagulated using heparin. Following anticoagulation, 


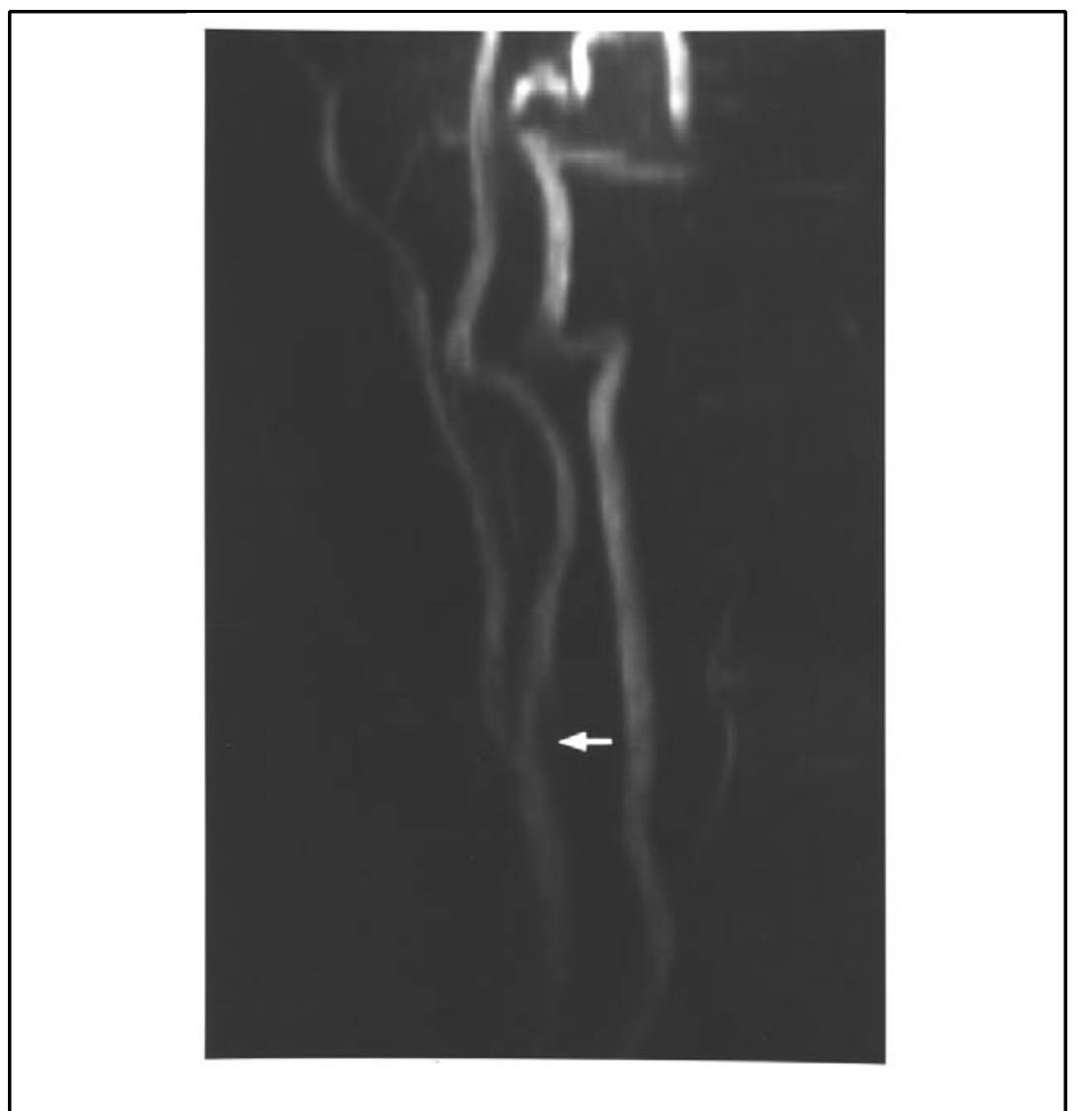

Fig 1. MRA of the neck revealing dissection of the internal carotid artery with a luminal flap

the patient reported improvement in her ptosis, conjunctival injection, and a modest reduction in her headache pain score (30\% to $40 \%)$.

At 3 months, a follow-up MRA revealed no interval change. The intimal flap at the origin of the ICA was again noted protruding into the lumen narrowing it approximately $65 \%$ involving a length of 8 $\mathrm{mm}$. There was normal flow distally within the ICA. A follow- up MRI brain also noted no interval changes but reveled punctuate high T2 signal areas within subcortical white matter and faint increased T2 signal within the peritrigonal white matter, which could be related to ischemic or demyelinating disease. A MRA of the brain at the same time was normal and did not reveal extension of the intimal tear into the brain. She maintained regular follow-up in our Pain Clinic at monthly intervals for symptomatic headache and low back pain. She was being managed conservatively on medications which included Tylenol \#3, clonazepam and Elavil. Her anticoagulation was still therapeutic and no further LESI were planned until her coagulation status normalized. creasing low back pain with radiculopathy. Considering that she was off anticoagulation and the dissection was stable, we performed LESI \#2 using $80 \mathrm{mg}$ methylprednisolone acetate in $2 \mathrm{ml}$ of $1 \%$ lidocaine hydrochloride with resolution of the radiculopathy pain. She had a third LESI again using the same combination of medications at 11 months. At 13 months, her radiculopathy pain had decreased but she complained of increasing neck pain. She had a superficial cervical plexus block performed using $10 \mathrm{~mL}$ of $0.25 \%$ bupivacaine with $80 \mathrm{mg}$ of methylprednisolone acetate with excellent neck pain relief. At 14 months, she presented with "mild" Horner's syndrome with a new finding of "facial burning". A stellate ganglion block using $8 \mathrm{~mL}$ of $0.25 \%$ bupivacaine and $80 \mathrm{mg}$ of methylprednisolone acetate was done with excellent pain relief. The stellate ganglion block was repeated again at 16 and 18 months, with resolution of her neck and facial pain. At the last follow-up at 36 months, her Horner's syndrome had returned full-blown and she continued to complain of moderate headaches, which were intermittent.

\section{DisCussion}

Spontaneous carotid artery dissection (SCAD) typically occurs without any antecedent history of trauma or tumor. However, in a prospective study by the Canadian Stroke Consortium (1), careful history taking in patients with so-called spontaneous dissection has invariably revealed minor trauma. It is likely that the etiology and pathogenesis of spontaneous carotid artery and vertebral artery dissections are multifactorial and that mechanical factors, such as sudden neck movements, and underlying arteriopathy have a role. The underlying arteriopathy may be transient and may be related to a recent infection, or the arteriopathy may be related to a genetic abnormality. Our patient had a history of incidental head "bumping" one week after receiving a LESI. If the trauma really contributed to the dissection is conjecture. The temporal relationship of the Horner's syndrome following the headache may implicate the initial trauma as being related to the dissection. Typically, following trauma, a hematoma forms and causes a dissection of the arterial wall that result in compression and collapse of the arterial lumen. Nerve damage occurs secondary to an interruption of blood flow and the compression that results from local inflammatory re- 
sponse to the hematoma. Injury to the superior cervical ganglion causes Horner's syndrome (2). SCAD is now recognized as the etiology of other cranial neuropathies including postcoital headache, scintillating scotomata (3), ipsilateral hypoglossal nerve palsy (4), multiple cervical neuropathies including cranial nerves 5 , 7, 9, 10 and 9, 10, 11, 12 (5). There is also at least one report of isolated abducens nerve palsy (6). The prognosis of patients with neurological sequelae from SCAD may be grave. Prospective studies have found SCAD to account for $2.5 \%$ to $10 \%$ of strokes. Therapy consists of short-term heparinization, followed by longer-term (6months or more) anticoagulation using coumadin until arterial recanalization has been demonstrated to occur.

Another disease considered in our differential diagnosis was Raeder's paratrigeminal syndrome (7) characterized by oculosympathetic paralysis including ptosis and miosis. This syndrome may be easily confused with Horner's syndrome. Raeder's syndrome is associated with head trauma, hypertension, vasculitis, migraine headache, parasellar mass lesions and internal carotid artery dissection. Trigeminal nerve irritation and preservation of facial sweating are noted. Our patient demonstrated unilateral loss of facial sweating, unlike those patients with Raeder's syndrome.

Patients with SCAD must be monitored at regular intervals by MRA, and when recanalization occurs, anticoagulation may be discontinued. However, in our patient the arterial lumen was fully patent and heparnizition was deemed merely prophylactic. The majority of patients will have restoration of blood flow within six months. Our patient has continued to be under our care and supervision for the past three years. Despite anticoagulation and no luminal flow restriction, her headaches have only improved to a moderate degree ( $50 \%$ to $60 \%$ ), and her photophobia has persisted.

We had a concern that systemic hypotension may lead to increase in the dissection and possible extension of the luminal flap. We were careful to prehydrate the patient with at least $500 \mathrm{ml}$ of isotonic fluids before we performed the second and third LESI. Whether this concern is true may need to be evaluated in other case reports in the future.

The role of stellate ganglion blocks in patients with SCAD has never been evalu- ated. In our case, the patient was relieved of both the headache and neck pain following the blocks. More case reports are needed to fully evaluate the role of sympathetic nerve blocks in such patients.

Dissection of the carotid artery is a dynamic process. The radiographic findings may change dramatically within a period of days or even hours. Although the radiographic appearance may worsen during the acute phase of dissection, about 90 percent of stenoses eventually resolve, two thirds of occlusions are recanalized, and one third of aneurysms decrease in size. This improvement takes place largely within the first two or three months after the dissection and is rare after six months. However, in our case the dissection has persisted in follow-up. Surgical or endovascular treatment should be reserved for patients who have persistent symptoms despite adequate anticoagulation. Surgical treatment consists of ligation of the carotid artery with an in situ or extracranial-to-intracranial bypass. These procedures can be associated with a high morbidity rate. Endovascular treatment, consisting of percutaneous balloon angioplasty and placement of one or more metallic stents, has gained favor over surgery as the initial therapy of choice once medical therapy fails $(8,9)$. However, the longterm results of carotid stenting are unknown, and the treatment of stent-related complications can be complex (10).

In summary, this case is fascinating in that a spontaneously occurring, and potentially life- threatening syndrome developed post- procedurally in an otherwise young healthy person seen in the pain clinic for lumbar radiculopathy. One week after receiving a completely uneventful LESI, she developed a sudden onset of intense headache accompanied by Horner's syndrome. The diagnosis of SCAD was expeditiously made, and the patient underwent treatment for it. Despite no carotid arterial luminal flow restriction and acceptable anticoagulation, the patient's headache and Horner's syndrome persisted for three years. Additionally, she became emotionally labile and her symptomatology worsened during periods of melancholy or tearfulness. She is slowly improving, and fortuitously for her, never developed irreversible neurological sequelae from SCAD. In patients with new onset headache and Horner's syndrome, detailed evaluation, including MRA, must be immediately undertaken to rule-out the possibility of SCAD. If there is a spontaneous dissection, appropriate therapy must be instituted immediately, since the potential for continued dissection or embolization remains significant until such therapy is instituted.

\begin{tabular}{l}
\hline Author Affiliation \\
Sukdeb Datta, MD \\
Director, Pain Management Center \\
VA Tennessee Valley Healthcare System \\
Nashville Campus \\
$131024^{\text {th }}$ Avenue South \\
Nashville, TN 37212 \\
E-mail: sukdeb@hotmail.com \\
Kenneth D. Candido, MD \\
Associate Professor of Anesthesiology \\
Northwestern University Hospital \\
251 East Huron St. \\
Chicago, IL 60611
\end{tabular}

\section{RefERENCES}

1. Norris JW, Beletsky V, Nadareishvili ZG. Sudden neck movement and cervical arterial dissection. CMAJ 2000; 163:38-40.

2. Resebraugh, CJ, Gnebed DJ, Dipette DJ. A case report of carotid artery dissection presenting as cluster headache. Am J Med 1997:10:418-419.

3. Ramadan NM, Tieljen GE Leirne SR et al. Scintillating scotomata associated with internal carotid artery dissection; present of three cases. Neurology 1991; 41:1084-1087.

4. Vighetto A, Lisovoske F, Revol A et al. Internal carotid artery dissection and ipsilateral hypoglossal nerve palsy. J Neural Neurosurg Psychiatry 1990; 53:530-531.

5. Panisset M, Eidelman BH. Case report: Multiple cervical neuropathy as a feature of internal carotid artery dissection. Stroke 1990; 21:141-147.

6. Maitland CG, Black JL, Smith WA. Abducens nerve palsy due to spontaneous dissection of internal carotid artery. Arch Neural 1983; 40:448-449.

7. Murnane M, Proano L. Raeder's paratrigeminal syndrome: A case report. Acad Emerg Med 1996; 3 (9): 864-867.

8. Liu AY, Paulsen RD, Marcellus ML et al. Long-term outcomes after carotid stent placement treatment of carotid artery dissection. Neurosurgery 1999; 45:1368-1374.

9. Malek AM, Higashida RT, Phatouros CC et al. Endovascular management of extracranial carotid artery dissection achieved using stent angioplasty. Am / Neuroradiol 2000; 21:1280-1292.

10. Schievink WI, Thompson RC, Lavine SD et al. Superficial temporal artery to middle cerebral artery bypass and external carotid reconstruction for carotid restenosis after angioplasty and stent placement. Mayo Clin Proc 2000; 75:1087-1090. 
